\title{
FATORES CRÍTICOS PARA O SUCESSO DO SEIS SIGMA: UM LEVANTAMENTO DO IMPACTO DO TEMPO DE EMPRESA E DO TREINAMENTO NA INDÚSTRIA ALIMENTÍCIA
}

\section{CRITICAL FACTORS FOR SIX SIGMA SUCCESS: A SURVEY OF THE IMPACT OF COMPANY TIME AND TRAINING IN THE FOOD INDUSTRY}

\author{
Everton Drohomeretski ${ }^{*, * *}$ Email: everton.drohomeretski@fae.edu \\ Sergio E. Gouvea da Costa* Email: $\underline{\text { s.gouvea@pucpr.br }}$ \\ Edson Pinheiro de Lima* Email: e.pinheiro@pucpr.br \\ Wesley Vieira da Silva* E-mail: wesley.vieira@pucpr.br \\ ${ }^{\star}$ FAE Centro Universitário (FAE), Curitiba, PR \\ *Pontifícia Universidade Católica do Paraná (PUC/PR), Curitiba, PR
}

\begin{abstract}
Resumo: A metodologia Seis Sigma apresenta um crescente interesse por parte de empresas dos diversos setores. Isso se dá, principalmente, em virtude dos seus resultados atrelados à redução de custos, aumento da confiabilidade e do padrão de qualidade. Porém, para que os resultados do Seis Sigma sejam alcançados e sustentados, faz-se necessária a gestão de alguns fatores críticos de sucesso (FCS) do Seis Sigma. O presente artigo tem por objetivo investigar os FCS para implantação da metodologia Seis Sigma e identificar quanto o tempo de empresa e o nível de formação Seis Sigma influenciam nos FCS. Para atingir o objetivo proposto foi realizada uma pesquisa de campo com 80 respondentes, com formação em Seis Sigma, de uma indústria alimentícia de grande porte localizada no Sul do Brasil. Os dados foram analisados por meio de estatística descritiva, teste de correlação, análise multivariada por meio da análise fatorial e análise de regressão múltipla. Com base na amostra pesquisada, os resultados evidenciaram que os FCS estudados apresentam uma contribuição significativa para o sucesso do programa Seis Sigma. Além disso, pode-se verificar que o tempo de empresa dos funcionários apresenta correlação positiva para o constructo treinamento, e o nível de formação em Seis Sigma apresenta correlação negativa com os constructos Treinamento, Comunicação e Revisão e Operação.
\end{abstract}

Palavras-chave: Seis Sigma. FCS. Indústria alimentícia. Survey. Análise multivariada.

Abstract: The Six Sigma methodology presents a growing interest from companies in different sectors. This happens mainly because of the results linked to cost savings, increased reliability and quality standards. However, for the Six Sigma results to be achieved and sustained it is necessary to manage some of the critical success factors (CSF) of Six Sigma. This paper aims to investigate the CSF for the implementation of Six Sigma methodology and identify how the time worked in the company and the level of training in Six Sigma influences the CSF. To achieve the proposed objective a field survey was conducted with 80 respondents, trained in Six Sigma, from a large food industry located in Southern Brazil. The data was analyzed using descriptive statistics, correlation test, multivariate analysis, factor analysis and multiple regression analysis. Based on the sample studied, the results showed that the CSF investigated had a significant contribution to the success of Six Sigma program. Moreover, it can be seen that the time worked in the company by employees is positively correlated to construct training and the level of training in Six Sigma has a negative correlation with the constructs Training, Communication, Review and Operation.

Keywords: Six Sigma. CSF. Food industry. Survey. Multivariate analysis.

Revista Produção Online, Florianópolis, SC, v. 16, n. 2, p. 475-498, abr.jun. 2016. 


\section{INTRODUÇÃO}

A busca pela eficiência nas etapas de concepção dos produtos, nos processos e na qualidade das operações, tem sido constantemente o enfoque das empresas como resposta aos desafios que têm surgido desde o início da era industrial. Com a economia global cada vez mais competitiva e com clientes mais exigentes e informados, oferecer produtos e serviços de melhor qualidade passou a ser um critério qualificador de mercado.

Com intuito de garantir a qualidade de seus produtos e a robustez dos seus processos, muitas empresas vêm adotando diversas ferramentas e metodologias de melhoria. Buscando atingir esse objetivo, principalmente a partir dos anos 50, as empresas japonesas apresentaram ganhos significativos. Logo as empresas americanas iniciaram um processo de busca a fim de se equipararem a elas ou até superá-las, e com estudos dos princípios de Shweart e Deming, no que diz respeito à importância em considerar as variações do processo e aos princípios estatísticos, nasce na Motorola a metodologia Seis Sigma, que logo foi disseminada para outras empresas, devido os ganhos atribuídos a ela.

Mahanti e Antony (2009) identificaram que a aplicação do Seis Sigma tem tornado possível que as empresas, além de produzirem com maior qualidade, melhorem a performance dos produtos, obtenham maior produtividade, reduzam custos e aumentem a satisfação dos clientes.

O Seis Sigma tem apresentado um aumento tanto na sua popularidade como na aceitação em indústrias em diversos países (NONTHALEERAK; HENDRY, 2008), e tal popularidade pode ser verificada com o crescimento no número de publicações científicas nos últimos anos (NONTHALEERAK; HENDRY, 2006, SCHROEDER et al., 2007). Um dos grandes destaques do Seis Sigma esta na possibilidade da obtenção de consideráveis ganhos financeiros (PINTO et al., 2014).

Para Kumar et al. (2011) o Seis Sigma pode ser implantado em diversos setores da economia e empresas de diversos portes, levando em consideração as devidas adaptações relacionadas ao contexto econômico e estratégico que a organização está inserida. No entanto, Nonthaleerak e Hendry (2008) destacam que 
empresas não industriais têm apresentado maior dificuldade no processo de implantação do Seis Sigma.

No Brasil, um setor que tem apresentado certo interesse na implantação do Seis Sigma é a indústria alimentícia. Parte desse interesse tem ligação com o aumento da concorrência e com o aumento na participação que o setor tem apresentado nos últimos anos na economia do país. Segundo a Associação Brasileira da Indústria da Alimentação (ABIA), a indústria alimentícia, englobando produtos alimentares e bebidas, tem uma participação de cerca de 9,3\% do Produto Interno Bruto (PIB) brasileiro e produção de $R \$ 383,3$ bilhões (ABIA, 2012).

Embora os ganhos apresentados com a implantação do Seis Sigma sejam significativos, alguns fatores críticos para o sucesso (FCS) devem ser levados em consideração para que a organização possa extrair da metodologia todos os seus benefícios. Dentre os FCS do Seis Sigma, pode-se destacar o envolvimento da alta administração, a infraestrutura organizacional, o treinamento em Seis Sigma, o tempo de empresa dos funcionários e a correta aplicação de ferramentas estatísticas (BRUN, 2011; DROHOMERETSKI et al., 2014; MARZAGÃO et al. (2014). Nesse sentido, o presente artigo tem como objetivo identificar o quanto o tempo de empresa e o nível de formação Seis Sigma influenciam nos FCS.

O artigo é organizado da seguinte forma: além da presente introdução, a seção 2 apresenta uma breve revisão da literatura, na seção 3 é descrito o método de pesquisa, na seção 4 os dados são apresentados e analisados, e as conclusões são relatadas na seção 5 .

\section{REVISÃO DA LITERATURA}

A presente seção visa apresentar o conceito do Seis Sigma, os seus benefícios, a sua aplicação e, principalmente, os fatores críticos de sucesso para a continuidade da metodologia. 


\subsection{A Metodologia Seis Sigma}

O Seis Sigma nasceu na Motorola, em 1987. Em 1988, quando a Motorola ganhou o Prêmio Nacional de Qualidade Malcolm Baldrige, o Seis Sigma passou a ser reconhecido como o responsável pelo sucesso da empresa. Entre as décadas de 80 e 90, a Motorola obteve ganhos de 2,2 bilhões de dólares com a aplicação do modelo (ECKES, 2001). Para Snee (2000), o Seis Sigma é uma estratégia de melhoria de negócios que busca identificar e eliminar as causas dos defeitos ou erros nos processos de negócios, concentrando-se nas atividades que são relevantes para os clientes. Moosa e Sajid (2010) complementam que o Seis Sigma é uma metodologia de melhoria no campo da Gestão da Qualidade Total. Brun (2011) destaca que o Seis Sigma melhora a satisfação dos clientes e reduz a variabilidade dos processos.

Nabhani e Shokri (2009) realizaram um estudo que objetivou identificar os resultados gerados pela metodologia DMAIC (método para resolução de problemas dividido em cinco etapas: Define, Measure, Analyze, Improve e Control), em uma indústria alimentícia. O estudo foi realizado na área de expedição da empresa e após a implantação, após a implantação do DMAIC o número de envios incorretos de mercadoria reduziu em aproximadamente $75 \%$.

De acordo com Näslund (2008), a implantação do Seis Sigma apresenta as seguintes características:

- entendimento do chão de fábrica das expectativas do projeto;

- liderança da alta administração;

- aplicação disciplinada do DMAIC;

- aplicação rápida do projeto (3-6 meses);

- definição clara dos resultados a serem alcançados;

- fornecimento de infraestrutura para aplicação das melhorias;

- foco no consumidor e no processo; e

- foco na abordagem estatística para melhoria.

Dentre as metodologias aplicadas no processo de implantação do Seis Sigma, a mais usual é o DMAIC. Para Andersson et al. (2006), o DMAIC é utilizado para a implantação do Seis Sigma em processos existentes. A referida metodologia 
tem a função de orientação à aplicação do modelo Seis Sigma, sendo dividida em 5 etapas. Vale destacar que algumas empresas optam por adotar o DMAIC mesmo sem ter o programa Seis Sigma implementado (PINTO et al., 2009). O Quadro 1, baseado em Kumar e Sosnoski (2009), apresenta as etapas do DMAIC com as respectivas ferramentas da qualidade a serem utilizadas.

Quadro 1 - Metodologia DMAIC
\begin{tabular}{|c|c|c|}
\hline № & Phase & Tools \\
\hline 1 & D - Define & Pareto Analysis; Project Charter. \\
\hline 2 & M - & Descriptive Statistics; Process Capability \\
& Measure & Analysis. \\
\hline 3 & A - Analyze & Detailed Process Map; Fish-Bone Diagram. \\
\hline 4 & I - Improve & Experimentation; New Process. \\
\hline 5 & C - Control & Statistical Process Control. \\
\hline
\end{tabular}

Fonte: Kumar e Sosnoski (2006)

Ao analisar os resultados de um estudo em uma indústria automotiva, Cleto e Quinteiro (2011) identificaram que a implantação de DMAIC na empresa estudada possibilitou que a análise do problema seguisse uma abordagem mais profunda e flexível. Além disso, permitiu um elevado conhecimento dos processos envolvidos na aplicação do DMAIC.

Em relação aos modelos de implantação, Chakravorty (2009) apresenta um modelo para implementação do Seis Sigma que seguem seis passos para a sua aplicação. Segundo o autor, no primeiro passo é necessário realizar uma análise da estratégia do mercado, o segundo passo é selecionar uma equipe de alto nível para a implantação das melhorias, o terceiro passo está voltado a selecionar as ferramentas, o quarto passo é identificar as oportunidades de melhoria, o quinto e sexto passos estão ligados à implantação e controle dos resultados alcançados.

Os significativos resultados alcançados, seguindo métodos adequados de implantação, são importantes fatores que justificam a implantação do Seis Sigma. Nesse sentido, Mahanti e Antony (2009) realizaram um survey em indústrias indianas de software, e identificaram que a aplicação do Seis Sigma tem possibilitado que as respectivas indústrias, além de produzirem softwares com maior qualidade, melhorem a performance dos produtos, obtenham maior produtividade, aumentem a satisfação dos clientes e reduzam custos.

Ao analisar as principais características do Seis Sigma e os seus fatores críticos para implementação em dez empresas brasileiras Pinto et. al. (2009) relatam

Revista Produção Online, Florianópolis, SC, v. 16, n. 2, p. 475-498, abr./jun. 2016. 
que a possibilidade de ganhos financeiros é potencializada após trinta e seis meses da sua implantação. Os autores relatam ainda que a redução de custos da nãoqualidade é o maior gerador de ganhos financeiros.

Su et al. (2006) organizaram os benefícios do Seis Sigma, conforme pode ser verificado abaixo:

- processo uniforme de saída dos produtos;

- redução de defeitos;

- redução de custos;

- melhoria da produtividade;

- mudança de cultura;

- satisfação do consumidor; e

- aumento do volume de vendas.

Kumar e Sosnoski (2009) analisaram que com a aplicação do Seis Sigma em uma indústria americana de ferramentas, a empresa estudada conseguiu reduzir em 20.000 o número de peças refugadas, gerando uma economia anual de aproximadamente $\bigcup \$ 12,500.00$. Assim, o Seis Sigma, além de melhorar a qualidade, reduz drasticamente os custos da organização.

Já em relação à manutenção do modelo Seis Sigma, Van Iwaarden et al. (2008) constataram que a manutenção do Seis Sigma nas organizações depende de uma cultura voltada para a qualidade na organização como um todo, pois, segundo os autores, as empresas que não utilizam ferramentas da qualidade antes da implantação do Seis Sigma acabam interrompendo o programa, principalmente pela necessidade de utilizar ferramentas estatísticas mais complexas.

Em um estudo que analisou o impacto do Seis Sigma nas áreas de decisão e nas prioridades competitivas da manufatura em indústrias localizadas no sul do Brasil, Drohomeretski et al. (2014) identificaram que o Seis Sigma tem impacto significativo no aumento da velocidade, flexibilidade, custo e qualidade.

Hung e Sung (2011) realizaram um estudo sobre a aplicação da metodologia Seis Sigma em uma indústria alimentícia e identificaram que com a sua aplicação a empresa conseguiu reduzir em 70\% o índice de defeitos em determinada linha de produção. Os autores destacam que a indústria alimentícia usualmente tem um nível de qualificação inferior a indústrias como a General Eletric e Motorola, que Revista Produção Online, Florianópolis, SC, v. 16, n. 2, p. 475-498, abr./jun. 2016. 
alcançaram resultados significativos com a implantação do Seis Sigma. Mais especificamente, na indústria alimentícia estudada os funcionários apresentavam um baixo nível de conhecimento sobre ferramentas da qualidade e estatística.

\subsection{Fatores críticos para o sucesso (FCS) do Seis Sigma}

Os componentes-chave para o sucesso na implantação do Seis Sigma estão relacionados ao envolvimento da alta administração, infraestrutura organizacional, treinamento e ferramentas estatísticas (HENDERSON; EVANS, 2000, VAN IWAARDEN et al. 2008, BRUN, 2011). Nesse mesmo sentido, Snee (1990) aponta que o Seis Sigma depende de uma combinação de oito características, incluindo resultados finais criados, liderança, ativa participação da alta administração, aplicação disciplinada do DMAIC, conclusão rápida do projeto (3-6 meses), definição clara dos objetivos, estabelecimento de formação e infraestrutura para os Master Black Belt, Black Belt e Green Belt, aplicação de estatística robusta focando resultados na melhoria dos processos e que impactem os clientes.

Moosa e Sajid (2010) realizaram um estudo que objetivou analisar os fatores críticos para o sucesso do Seis Sigma. Para os autores, o sucesso e o fracasso da maioria dos programas Seis Sigma, em grande parte, dependem de sua execução e não do seu conteúdo. Os autores relatam que apenas um quarto das empresas que implantam o programa conseguem atingir os resultados esperados. Exemplo disso é retratado por Hindo e Grow (2007), os quais relataram que empresas como $3 \mathrm{M} \mathrm{e}$ Home Depot não ficaram satisfeitas com as resultados gerados após a implementação da metodologia Seis Sigma.

Moosa e Sajid (2010) destacam que as equipes Seis Sigma devem ser treinadas para garantir a competência adequada na utilização de várias ferramentas e técnicas necessárias; se as equipes pararem de funcionar, o programa Seis Sigma para. Ainda, Moosa e Sajid (2010) destacam que o sucesso na implantação dos Seis Sigma requer uma boa visão das principais ferramentas de gestão; estratégias apropriadas com base em experiências; práticas e a sólida formação dos envolvidos para o uso adequado de ferramentas avançadas de estatística; aplicação dos processos do DMAIC; coordenação eficaz através de uma gestão adequada do 
projeto -principalmente nos 2 primeiros anos de implantação; liderança que exige responsabilização efetiva; e motivação e trabalho em equipe de gestores. Yi-Zhong et al. (2011) identificaram por meio de um survey em indústrias chinesas que o fator liderança tem um efeito significativo nos projetos Seis Sigma.

Shahin (2006) organizou os principais FCS. Para isso, o autor destacou que entre os fatores liderança e compromisso da alta administração, ligando o Seis Sigma à estratégia de negócios, apoiando a infraestrutura organizacional, treinamento e a compreensão da metodologia Seis Sigma são os fatores mais importantes, qualquer organização deve considerar em seu projeto Seis Sigma.

Em um estudo com duas empresas multinacionais, Santos e Martins (2010) investigaram como o efeito dos investimentos em Seis Sigma impactam na efetividade do programa. Os autores relatam que resultados sustentáveis obtidos por meio do Seis Sigma dificilmente acontecem no curto prazo, dessa forma, o imediatismo pode ser um FCS.

Rodrigues e Werner (2011) realizaram 11 estudos de caso em empresas brasileiras localizadas no Rio Grande do Sul. Os autores analisaram as atribuições da gestão de pessoal nos problemas presentes no processo de implantação do Seis Sigma, e identificaram que, nas empresas pesquisadas, fatores como liderança, comunicação e treinamento foram os principais no processo de implantação do Seis Sigma.

Zailani e Sasthriyar (2011) identificaram por meio da literatura alguns fatores críticos para o sucesso do Seis Sigma, dentre os quais os autores destacam o envolvimento da alta administração e do comitê organizador, a mudança cultural, a infraestrutura organizacional, o treinamento, as habilidades dos gestores dos projetos, sistemática de priorização, seleção, acompanhamento e revisão de projetos, entendimento da metodologia, ferramentas e técnicas Seis Sigma. Trad (2006) identificou, por meio de um survey em indústrias brasileiras, 8 FCS da metodologia Seis Sigma. O Quadro 1 ilustra os FCS identificados por Trad (2006). 
Quadro 1 - FCS do Seis Sigma

\begin{tabular}{|c|c|}
\hline & Fator \\
\hline 1 & Iniciativas prévias de qualidade \\
\hline 2 & Liderança \\
\hline 3 & Processo gerencial \\
\hline 4 & Perfil dos Black Belts \\
\hline 5 & Treinamento \\
\hline 6 & Projetos \\
\hline 7 & Equipes de projetos \\
\hline 8 & Comunicação e revisão \\
\hline
\end{tabular}

Já Sivakumar e Muthusamy (2011) realizaram um survey em indústrias da Malásia e identificaram que os FCS para as empresas pesquisadas são: a) comprometimento da alta administração; b) absorção da metodologia; c) projeto e processo de avaliação; d) formação e sensibilização dos funcionários.

Em uma meta-análise sobre os FCS do Seis Sigma, Marzagão et al. (2014) identificaram que os FCS mais apontados pela literatura pesquisada foram: cultura da qualidade; envolvimento dos funcionários; seleção de projetos; e treinamento e aprendizado.

\section{MÉTODO DE PESQUISA}

Em relação ao delineamento da pesquisa, pode-se caracterizá-la como um estudo de caráter predominantemente quantitativo. Esta abordagem é eleita para viabilizar a verificação dos principais FCS do Seis Sigma. O estudo tem um corte transversal, pois os dados foram coletados em um única etapa e a unidade de análise dos funcionários com formação em Master Black Belt (MBB), Black Belt BB), Green Belt (GB) e Yellow Belt (YB), além da Alta Gerência ( $A G)$ de uma indústria alimentícia brasileira de grande porte.

Em relação ao objetivo da pesquisa, o presente estudo trata-se de uma pesquisa explicativa. O método de pesquisa escolhido foi o survey. O survey é um método de pesquisa de abordagem quantitativa na qual a coleta de dados sobre uma amostra deverá fornecer dados representativos da população de interesse (FORZA, 2002, MALHOTRA; GROVER, 1998). 
A presente pesquisa adaptou a estrutura metodológica proposta por Forza (2002), a qual é desmembrada em cinco etapas: 1) ligação com o referencial teórico; 2) projeto do survey; 3) coleta de dados para teste da teoria; 4) análise dos dados; e 5) geração de relatório.

A amostragem foi não-probabilística, do tipo intencional por julgamento. Com um total de 140 funcionários com formação de GBs e YBs, estimou-se um número mínimo de 49 respondentes, considerando 95\% de nível de confiança e 5\% de erro. Para os funcionários GBs e YBs obteve-se um retorno de 63 respondentes, adicionando os $17 \mathrm{MBBs}$, BBs, e AGs, o que totalizou 80 respondentes.

Com a utilização do software SPSS, versão 9.0, a análise da confiabilidade das respostas foi realizada usando a análise fatorial, uma técnica estatística multivariada. Para tanto, foram calculadas medidas de confiabilidade das respostas coletadas e teste de significância não-paramétrico de Spearman, para a comparação das variáveis dependentes com as variáveis independentes.

\subsection{Procedimento para coleta de dados}

O estudo se enquadra como um levantamento de campo ou survey, por direcionar os esforços para a identificação dos FCS, com base na percepção dos funcionários com formação específica em Seis Sigma de uma indústria alimentícia, dessa forma, esse enquadramento permite a utilização de técnicas estatísticas para a apresentação e interpretação de dados, conforme será apresentado em seções futuras. Consoante a isto, o estudo constitui-se de dados predominantemente primários, os quais se referem à pesquisa de campo a ser realizada junto à empresa selecionada e que compõem a base de dados sujeita ao tratamento estatístico. Já os dados secundários envolvem a literatura sobre o tema, compilada para contextualizar e dirigir o estudo.

Desta forma, os procedimentos de coleta de dados abrangem a utilização de um questionário dirigido aos colaboradores da empresa que tiveram formação em Seis Sigma e que participam ou já participaram de projetos Seis Sigma dentro da empresa estudada. 
O questionário contém um total de 40 questões e foi estruturado em quatro blocos:

- O Bloco 1 identifica a função, tempo de empresa, qual a formação do respondente em Seis Sigma e o tipo de projeto Seis Sigma que o respondente já participou (produtividade, qualidade, estoque, entre outros);

- O Bloco 2 identifica a importância do programa Seis Sigma na dimensão Cultural, que inclui 3 questões referentes à compressão do Seis Sigma, conhecimento do programa Seis Sigma e o quanto o respondente acredita nesta metodologia;

- O Bloco 3 inclui perguntas relacionadas ao quanto a Liderança influencia no sucesso do Seis Sigma. São 5 perguntas, de modo que os respondentes computaram a importância relacionada à alta administração relativo ao grau de persistência, comunicação das políticas e expectativas de desempenho, compromisso da alta administração com a melhoria contínua, disponibilização de recursos, estímulo e suporte para os funcionários;

- O Bloco 4 apresenta 5 questões, que se relacionam à dimensão Treinamento, essas buscaram identificar o grau de importância que treinamentos relacionados à gestão de projetos e utilização de ferramentas da qualidade e estatística têm para o sucesso do Seis Sigma.

Cada pergunta representa uma variável de análise. Como são 40 questões, tem-se o mesmo número de variáveis. Para facilitar a tabulação dos dados, foi desenvolvido um código para cada uma delas.

O Quadro 2 apresenta a síntese dos instrumentos de coleta de dados e como esses foram analisados.

Para a pesquisa em questão, utilizaram-se escalas nominais (múltipla escolha) e escalas intervalares (escala Likert de sete pontos). No primeiro bloco, há questões de múltipla escolha. Nos blocos 2, 3 e 4, utilizou-se uma escala de importância de 7 pontos (1 - Não Importante, 4 - Neutro até 7 - Muito Importante).

Os questionários foram enviados, em um primeiro momento, por e-mail para os setenta e oito respondentes com o perfil GB e YB e dezessete profissionais de Melhoria Contínua. Após aproximadamente trinta dias, o convite para o 
preenchimento do questionário foi reenviado, como parte da estratégia para estimular maior participação.

Quadro 2 - Síntese dos instrumentos de coleta e análise de dados

\begin{tabular}{|c|c|c|}
\hline Bloco & Tema & Método de Análise \\
\hline 1 & Identificação do respondente & $\begin{array}{lll}\text { Estatística descritiva/Distribuição de } \\
\text { Frequência }\end{array}$ \\
\hline 2 & $\begin{array}{l}\text { Importância do programa Seis Sigma } \\
\text { (dimensão Cultural) }\end{array}$ & Análise da média/ Análise multivariada \\
\hline 3 & $\begin{array}{l}\text { Importância do programa Seis Sigma } \\
\text { (dimensão Liderança) }\end{array}$ & Análise da média/ Análise multivariada \\
\hline 4 & $\begin{array}{l}\text { Importância do programa Seis Sigma } \\
\text { (dimensão Treinamento) }\end{array}$ & Análise da média/ Análise multivariada \\
\hline 5 & $\begin{array}{l}\text { Importância do programa Seis Sigma } \\
\text { (dimensão Comunicação e Revisão) }\end{array}$ & Análise da média/ Análise multivariada \\
\hline 6 & $\begin{array}{l}\text { Impacto percebido no desempenho } \\
\text { operacional }\end{array}$ & Análise da média/ Análise multivariada \\
\hline 7 & Cultura da Qualidade & Análise da média/ Análise multivariada \\
\hline 8 & $\begin{array}{l}\text { Nível de sucesso da implantação do } \\
\text { Seis Sigma no setor em que atua }\end{array}$ & Distribuição de frequência \\
\hline
\end{tabular}

Fonte: Os autores (2014)

A pesquisa foi reenviada novamente para os profissionais que não responderam ao questionário na primeira solicitação, havendo um índice de retorno de $19 \%$ dos respondentes GB e YB, e um índice de retorno de $79 \%$ para os profissionais de Melhoria Contínua, totalizando um índice de retorno de $82 \%$ para estes profissionais e um índice total de retorno de $27 \%$ dos respondentes GB e YB.

\subsection{Hipóteses da pesquisa}

A partir da revisão bibliográfica, definiram-se os fatores como críticos para o sucesso da implantação da metodologia Seis Sigma nas organizações e foram levantadas duas hipóteses, de forma a identificar o quanto o tempo de empresa e o nível de formação em Seis Sigma influenciam nos FCS pesquisados.

A primeira hipótese testada foi:

- H1: tempo de empresa tem influência direta nos FCS.

Han e Lee (2002) defendem que o treinamento, principalmente do BB influencia positivamente na implantação do Seis Sigma nas organizações. O treinamento aplicado ao programa inclui o de liderança para profissionais envolvidos, de ferramentas analíticas e estatísticas, de uso de software estatístico, conceitos de 
qualidade, de método para solução de problemas, de gerenciamento de projetos e de trabalho em equipe (TRAD, 2006).

Já a segunda hipótese foi:

- H2: nível de formação em Seis Sigma tem influência direta no FCS.

A metodologia Seis Sigma tem como um dos requisitos a necessidade da implementação de programas de treinamento. O processo de qualificação é para variados níveis, desde básicos até avançados para os Master Black Belts e Black Belts. Rodrigues e Werner (2011) identificaram que o treinamento é um importante fator crítico para o sucesso do Seis Sigma. Trad e Maximiano (2009) destacam que a qualificação dos funcionários é uma FCS importante e que deve envolver além do treinamento de ferramentas estatística e da qualidade, questões voltadas à liderança e solução de problemas.

O Quadro 3 apresenta, de forma sucinta, os dois fatores críticos para o sucesso da implantação da metodologia Seis Sigma analisados com as respectivas hipóteses.

Quadro 3 - Proposta de fatores críticos de sucesso para implantação do Seis Sigma

\begin{tabular}{|l|l|}
\hline Fatores propostos & Hipóteses \\
\hline Correlação com o tempo de empresa & $\begin{array}{l}\text { H1: o tempo de empresa tem influência direta } \\
\text { com os FCS. }\end{array}$ \\
\hline Correlação com a formação em Seis Sigma & $\begin{array}{l}\text { H2: o nível de formação em Seis Sigma tem } \\
\text { influência direta nos FCS. }\end{array}$ \\
\hline
\end{tabular}

Fonte: Os autores (2014)

Para o teste das hipóteses $\mathrm{H} 1$ e $\mathrm{H} 2$ foi utilizada, incialmente, a análise fatorial. Após isso, foi realizado o teste de regressão linear múltipla para a comparação das variáveis dependentes com as variáveis independentes (tempo de empresa e formação Seis Sigma). A Figura 1 ilustra o modelo conceitual para $\mathrm{H} 1$ e $\mathrm{H} 2$. 
Figura 1 - Modelo conceitual para a $\mathrm{H} 1$ e $\mathrm{H} 2$

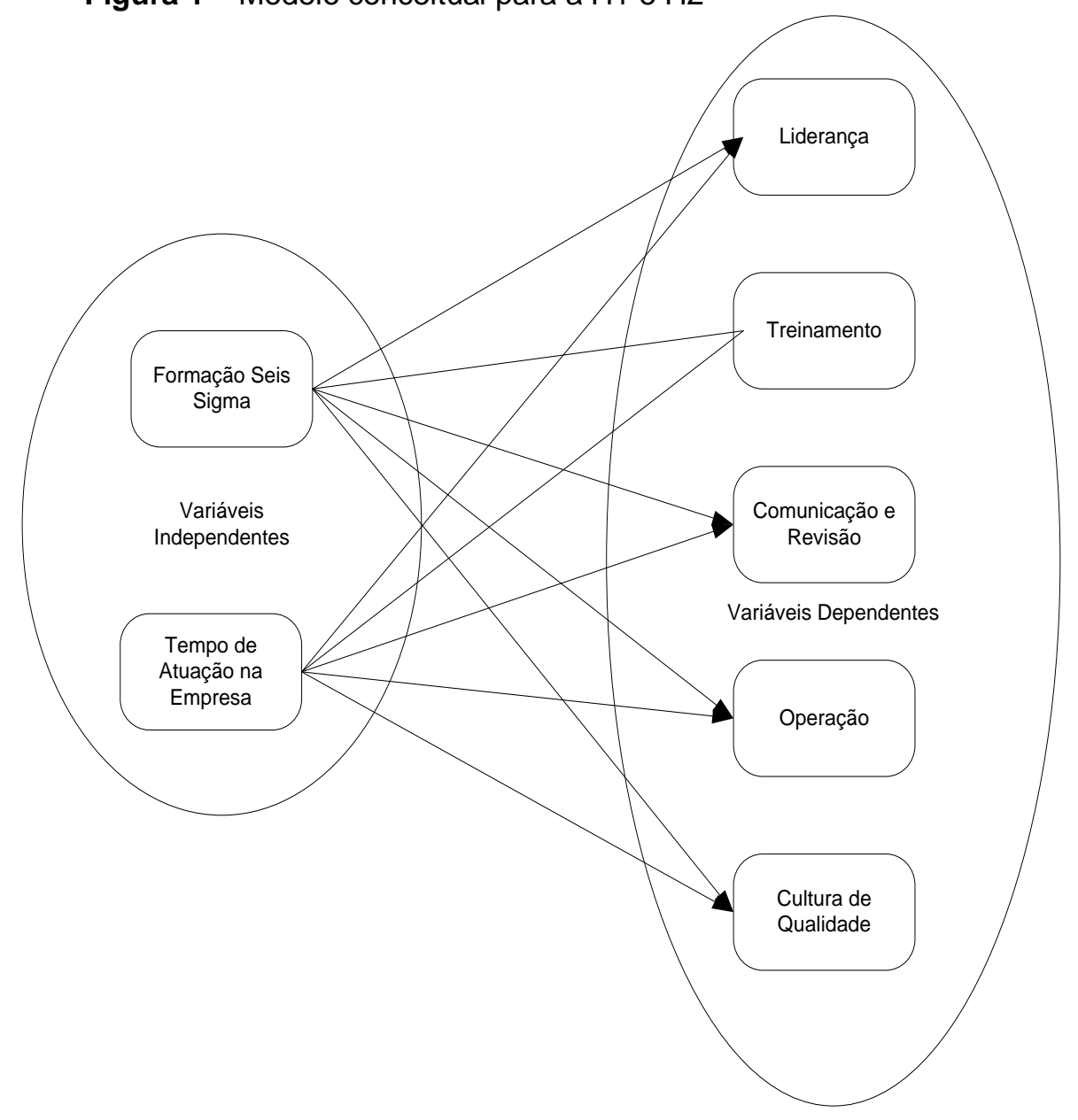

Fonte: Os autores (2014)

O processo de análise de dados se dará por meio de análise multivariada do teste das duas hipóteses.

\section{COLETA E ANÁLISE DOS DADOS}

A amostra coletada é composta, em sua maioria, por $43,8 \%$ dos respondentes, sendo YBs e $32,5 \%$ de GBs. A alta participação desses profissionais é importante para os objetivos da pesquisa e contribui para que haja uma representatividade das percepções sobre o programa.

Do total de respondentes 73,8\% possuem nível superior e 17,5\% têm nível técnico. Esses dois grupos somados são responsáveis por $91,2 \%$ dos questionários respondidos. Em relação ao tempo de empresa, $46,3 \%$ dos respondentes trabalham há mais de oito anos na empresa, e outros $33,8 \%$ trabalham há menos de dois anos.

Revista Produção Online, Florianópolis, SC, v. 16, n. 2, p. 475-498, abr./jun. 2016. 
Para investigar os tipos de projetos Seis Sigma em que os respondentes já estiveram envolvidos, os mesmos puderam selecionar quantas opções fossem pertinentes. Os tipos de projetos estão listados na Tabela 1, em ordem de citações. O tipo de projeto onde houve a maior participação dos respondentes é para redução de erros, falhas e geração de refugo, citados por $67,5 \%$ deles, seguido pelos projetos para o aumento de produtividade, apontado por $61,3 \%$ e projetos para a redução de custos, citado por 57,5\%. Seguindo a ordem decrescente, os projetos citados são melhoria da qualidade de produtos e serviços, redução do tempo de processamento, redução de inventário e melhoria da satisfação de clientes. $O$ menos citado foi para o desenvolvimento de novos produtos, por $15,0 \%$ dos respondentes.

Tabela 1 - Projetos em que os respondentes estiveram envolvidos

\begin{tabular}{lcc}
\hline \multicolumn{1}{c}{ TIPO DE PROJETO } & FREQUENNCIA & \% DO TOTAL \\
\hline Redução de erros/falhas/defeitos/refugo & 54 & 67,5 \\
Aumento de produtividade & 49 & 61,3 \\
Redução de custo & 46 & 57,5 \\
Melhoria da qualidade de produtos e serviços & 24 & 30,0 \\
Redução do tempo de processamento & 21 & 26,3 \\
Redução de inventário & 19 & 23,8 \\
Melhoria da satisfação de clientes & 18 & 22,5 \\
Desenvolvimento de novos produtos & 12 & 15,0 \\
Outros tipos & 21 & 26,3 \\
Total de respondentes & 80 & \\
\hline
\end{tabular}

Fonte: Os autores (2014)

Esses resultados vão ao encontro dos levantados na literatura mapeada. Redução de erros, falhas e defeitos e melhoria da qualidade de produtos e serviços são os principais objetivos propostos pela metodologia Seis Sigma (HARRY; SCHROEDER, 2000). Além disso, outro aspecto encontrado na literatura que justifica esse resultado é o fato de o custo da baixa qualidade representar $50 \%$ do orçamento nas empresas de serviços e de $10 \%$ a $20 \%$ nas empresas de operações fabris (PANDE et al., 2001). Outros tipos foram citados e representam 26,3\% dos projetos: redução de downtime, automações de processos manuais, redução de ruptura, redução do consumo de energia elétrica, logística, redução de geração de resíduos e implementação de tecnologia de informação. 


\subsection{Análise fatorial}

No processo da análise fatorial, seguiu-se as orientações de Hair et al. (2005), analisando a comunalidade, o teste de Kaiser-Meyer-Olkin (KMO), a confiabilidade e a variância explicada. $\mathrm{Na}$ primeira fase dos seis constructos analisados, somente o primeiro (Cultural) teve uma variável excluída por apresentar um Alpha de Conbrach abaixo de 0,6. O teste KMO é uma medida de adequação amostral que procura detectar se as correlações parciais entre as variáveis são pequenas e, neste caso, conforme a Tabela 2 todos os constructos apresentaram KMO de no mínimo 0,5. O que demonstra estar dentro dos parâmetros apresentados por Hair et al. (2005).

Outro teste realizado na análise fatorial é o de confiabilidade. Para Devellis (2003), a confiabilidade é a proporção da variância que pode ser atribuída ao verdadeiro valor da variável latente. Para isso, utilizou-se do cálculo do coeficiente Alpha de Cronbach. Como pode ser evidenciado na Tabela 2, o coeficiente Alfa de Cronbach foi superior a $0,6(\mathrm{CRONBACH}, 1951)$ para todos os constructos multiitens.

Tabela 2 - Síntese da Análise Fatorial

\begin{tabular}{ccccc}
\hline Constructos & Número de itens & Alfa de Cronbach & KMO & $\begin{array}{c}\text { Variância } \\
\text { Explicada \% }\end{array}$ \\
\hline Cultural & 2 & 0,628 & 0,500 & 73,841 \\
Liderança & 5 & 0,788 & 0,799 & 55,665 \\
Treinamento & 5 & 0,834 & 0,823 & 60,767 \\
Comunicação e & 5 & 0,689 & 0,692 & 46,627 \\
Revisão & 13 & & & \\
Operação & 5 & 0,899 & 0,858 & 70,913 \\
Cultura da Qualidade & & 0,692 & 0,635 & 70,167 \\
\hline
\end{tabular}

Fonte: Os autores (2014)

Tomando como base os constructos com maior e menor valor do KMO, podese verificar que o constructo Operação tem ajuste de adequação aos dados (KMO = 0,858 ) de aproximadamente $86 \%$ de uma escala de 0 a $100 \%$, já o constructo Cultural tem ajuste de adequação aos dados $(\mathrm{KMO}=0,500)$ de $50 \%$. Em relação à análise de confiabilidade, o constructo Operação apresentou um Alpha de Cronbach de 0,899 , o que indica um nível de aproximadamente $90 \%$, ou seja, suas medidas concordariam em $90 \%$ das vezes, já para o constructo Cultural apresentou um Alpha

Revista Produção Online, Florianópolis, SC, v. 16, n. 2, p. 475-498, abr./jun. 2016. 
de Cronbach de 0,628, o que indica um nível de aproximadamente 63\%, ou seja, suas medidas concordariam em $63 \%$ das vezes.

Por sua vez, o constructo Cultural teve o maior poder de explicação da variância $(73,84 \%)$, já o constructo com menor poder de explicação da variância foi o de Comunicação e Revisão (46,63\%).

Após a validação da análise fatorial realizou-se o teste de normalidade. Como o número de respondentes foi superior a 30 (total 80 respondentes), optou-se pela análise de normalidade Kolmogorov-Smirnov (KS). Como se pode verificar na Tabela 3, o único constructo que apresentou um grau de significância acima de 0,05 foi o de operação.

Tabela 3 - Teste de Normalidade KS

\begin{tabular}{llrrrrrr}
\hline & & & & \multicolumn{3}{c}{ Comunicação } & \multicolumn{2}{c}{$\begin{array}{c}\text { Cultura da } \\
\text { Qualidade }\end{array}$} \\
\hline $\mathrm{N}$ & & Cultural & Liderança & Treinamento & e revisão & Operação & 80 \\
Normal & Média & 80 & 80 & 80 & 80 & 80 & 80 \\
Parâmetros a,b & Desvio Padrão & 1,00000000 & 1,00000000 & 1,00000000 & 1,00000000 & 0,49931555 & 0,52340232 \\
Limite Superior & Absoluto & 0,177 & 0,171 & 0,197 & 0,160 & 0,112 & 0,224 \\
Diferenças & Positiva & 0,153 & 0,171 & 0,197 & 0,160 & 0,108 & 0,224 \\
& Negativa & $-0,177$ & $-0,161$ & $-0,157$ & $-0,157$ & $-0,112$ & $-0,176$ \\
Kolmogorov-Smirnov Z & & 1,582 & 1,528 & 1,766 & 1,427 & 0,998 & 2,001 \\
Asymp. Sig. (2-tailed) & & 0,013 & 0,019 & 0,004 & 0,034 & 0,272 & 0,001 \\
\hline
\end{tabular}

Fonte: Os autores (2014)

Baseado no teste de normalidade, aplicou-se um teste não-paramétrico, no caso o teste de Spearman. Ele é adequado para a análise de dados com tamanho de amostra maior de 10 e para dados distribuídos não-normalmente. Com base no modelo desenvolvido (Figura 1), utilizou-se como fatores independentes o Tempo de Empresa e o Tipo de Formação Seis Sigma (MBB, BB, YB ou GB), e como fatores dependentes os seis processados na análise fatorial.

Pode-se observar (Tabela 4) que o Tempo de Empresa apresenta correlação significativa com o constructo Treinamento, representando uma fraca correlação, e conclui-se que o Tempo de Empresa tem correlação de 0,266 (26,60\%) com o constructo Treinamento.

Já em relação ao fator Formação Seis Sigma, os constructos que apresentaram correlação significativa foram o Treinamento, Comunicação e Revisão e Operação. Em relação ao constructo Treinamento apresentou uma correlação negativa de - 0,392 (- 39,20\%). O constructo Comunicação e Revisão apresentou Revista Produção Online, Florianópolis, SC, v. 16, n. 2, p. 475-498, abr./jun. 2016. 
correlação negativa de $-0,309$ (- 30,09\%). Por fim, o constructo operação teve uma correlação negativa de $-0,270(-27,00 \%)$.

Tabela 4 - Estimativa da Correlação de Spearman

\begin{tabular}{|c|c|c|c|c|c|c|}
\hline & \multicolumn{2}{|c|}{ Cultural } & \multicolumn{2}{|c|}{ Liderança } & \multicolumn{2}{|c|}{ Treinamento } \\
\hline & Correlação & Sig. & Correlação & Sig. & Correlação & Sig. \\
\hline \multirow{4}{*}{$\begin{array}{c}\text { Tempo de Empresa } \\
\text { Formação Seis } \\
\text { Sigma }\end{array}$} & $-0,056$ & 0,623 & $-0,071$ & 0,534 & $0,266^{\star}$ & 0,017 \\
\hline & 0,110 & 0,332 & 0,141 & 0,212 & $-0,392^{\star}$ & 0,000 \\
\hline & \multicolumn{2}{|c|}{$\begin{array}{l}\text { Comunicação e } \\
\text { Revisão }\end{array}$} & \multicolumn{2}{|c|}{ Operação } & \multicolumn{2}{|c|}{ Cultura da Qualidade } \\
\hline & Correlação & Sig. & Correlação & Sig. & Correlação & Sig. \\
\hline $\begin{array}{l}\text { Tempo de Empresa } \\
\text { Formação Seis }\end{array}$ & 0,188 & 0,095 & 0,134 & 0,237 & 0,061 & 0,589 \\
\hline Sigma & $-0,309^{\star \star}$ & 0,005 & $-0,270^{*}$ & 0,016 & 0,078 & 0,490 \\
\hline
\end{tabular}

Fonte: Os autores (2014)

Os outros fatores não apresentaram significância com os dois fatores independentes analisados. Dessa forma, não foram discutidos.

\subsection{Regressão linear múltipla}

De forma a aprofundar os resultados da análise de correlação (Tabela 4), realizou-se também a análise de regressão linear múltipla, que é demonstrada a seguir na Tabela 5 . Um dos requisitos para a realização da regressão linear múltipla é o teste de multicolinearidade.

Para analisar a correlação entre os previsores no modelo foram analisados os valores VIF (Variance Inflation Factor) e Tolerância (inverso do VIF). Baseado em Hair Jr. et al. (2005), valores acima de 0,10 para Tolerância e abaixo de 10 para o VIF. Os dados testados na presente pesquisa apresentaram um valor de Tolerância de 0,814 e um VIF de 1,228, ou seja, dentro dos parâmetros esperados, relevando a não existência de problemas graves de multicolinearidade.

Com base nos dados obtidos (Tabela 5), pode-se observar que nenhum constructo apresentou significância estatística menor que 0,05, o que representa que a variáveis não apresentam regressão com nenhum dos seis constructos. 
Analisando os betas, os dois fatores apresentaram betas baixos, sendo que a formação Seis Sigma apresentou betas um pouco melhores. Já analisando o $\mathrm{R}^{2}$ ajustado em relação ao $\mathrm{R}^{2}$ que somente o constructo Cultural apresenta uma boa proximidade entre $0 \mathrm{R}^{2}$ e $\circ \mathrm{R}^{2}$ ajustado, o que demonstra um alto poder de generalização para esse constructo.

Já a análise da estatística Durbin-Watson foi realizada para analisar a interdependência dos erros. Para Field (2009), os valores devem estar entre 0 e 4, com 2 significando que os resíduos não são correlacionados. Como se pode verificar na Tabela 5, todos os resultados da estatística Durbin-Watson ficaram próximos de 2, demonstrando que os resíduos não são correlacionados.

Tabela 5 - Resultados da Análise de Regressão Linear Múltipla - betas, $\mathrm{R}^{2}$ e $\mathrm{R}^{2}$ ajustado

\begin{tabular}{lrrrrrr}
\hline & Cultural & Liderança & Treinamento & $\begin{array}{c}\text { Comunicação } \\
\text { e Revisão }\end{array}$ & Operação & $\begin{array}{r}\text { Cultura da } \\
\text { Qualidade }\end{array}$ \\
\hline $\begin{array}{l}\text { Tempo de Empresa } \\
\text { Formação Seis }\end{array}$ & $-0,072$ & $-0,013$ & 0,058 & 0,076 & $-0,083$ & 0,089 \\
Sigma & 0,124 & 0,185 & $-0,233$ & $-0,202$ & $-0,182$ & $-0,051$ \\
$\mathrm{R}^{2}$ & 0,028 & 0,037 & 0,070 & 0,060 & 0,027 & 0,014 \\
$\mathrm{R}^{2}$ ajustado & 0,030 & 0,012 & 0,045 & 0,036 & 0,002 & $-0,011$ \\
Sig. & 0,334 & 0,238 & 0,062 & 0,092 & 0,062 & 0,575 \\
Durbin-Watson & 1,712 & 1,996 & 2,208 & 2,175 & 1,8432 & 2,043 \\
\hline
\end{tabular}

Fonte: Os autores (2014)

Outro quesito analisado na regressão linear múltipla é o teste de normalidade dos resíduos. A Tabela 6 ilustra os resultados do teste KS para os resíduos.

Tabela 6 - Teste de Normalidade dos Resíduos

\begin{tabular}{|c|c|c|c|c|c|c|}
\hline \multicolumn{7}{|c|}{ Comunicação e } \\
\hline & Cultural & Liderança & Treinamento & Revisão & Operação & Cultura da Qualidade \\
\hline KS & 0,000 & 0,005 & 0,000 & 0,001 & 0,013 & 0,000 \\
\hline
\end{tabular}

Fonte: Os autores (2014)

Pode-se verificar com base na Tabela 6 que nenhum constructo apresentou significância maior que 0,05 , desta forma se pode afirmar que os resíduos não apresentam uma distribuição normal.

Revista Produção Online, Florianópolis, SC, v. 16, n. 2, p. 475-498, abr./jun. 2016. 


\subsection{Análise das hipóteses}

Como parte dos requisitos para a regressão linear múltipla não foram cumpridos, os resultados do teste das hipóteses $\mathrm{H} 1$ e $\mathrm{H} 2$ foram analisados com base no resultados do teste de Spearman (Quadro 4).

Quadro 4 - Teste da $\mathrm{H} 1$ e $\mathrm{H} 2$

\begin{tabular}{|l|l|l|}
\hline I & Descrição da Hipótese & Hipóteses \\
\hline H1 & O tempo de empresa tem influência direta com os FCS. & Corroborada parcialmente \\
\hline H2 & $\begin{array}{l}\text { O nível de formação sem Seis Sigma tem influência direta } \\
\text { nos FCS. }\end{array}$ & Corroborada parcialmente \\
\hline
\end{tabular}

Fonte: Os autores (2014)

A H1 foi confirmada parcialmente, pois apresentou significância somente para o constructo Treinamento. Apresentando uma correlação positiva entre o tempo de empresa e a contribuição que o treinamento traz para o sucesso da implantação do Seis Sigma. No entanto, foi possível identificar que o tempo de empresa, embora não seja um FCS de grande destaque na literatura pesquisada, apresentou-se nesta pesquisa como um fator influente para o sucesso na implantação e sustentação da metodologia Seis Sigma nas empresas, no caso da presente pesquisa, a indústria alimentícia.

Por fim, a $\mathrm{H} 2$ também foi confirmada parcialmente. $\mathrm{O}$ teste demonstrou significância para a correlação negativa do nível de Formação Seis Sigma com o constructo Treinamento, Comunicação e Revisão e Operação. Com isso, pode-se verificar que o nível de formação Seis Sigma apresenta uma ligação negativa com estes três constructos. Ou seja, com base na percepção dos respondentes esses três fatores sucesso não tem relação direta com o nível de formação Seis Sigma. Esse resultado vai em direção contrária aos encontrados por Rodrigues e Werner (2011) e Trad e Maximiano (2009), no entanto, cabe destacar que as pesquisas realizados pelo autores citados foram realizadas em segmentos de mercado diferentes. 


\section{CONSIDERAÇÕES FINAIS}

Esta pesquisa teve como principal objetivo investigar o quanto o tempo de empresa e o nível de formação de Seis Sigma influenciam os fatores críticos de sucesso investigados. Os dados foram coletados por meio de uma pesquisa de campo realizada com profissionais ligados ao programa Seis Sigma de uma empresa alimentícia de grande porte, localizada no Sul do Brasil. Foram analisados dois aspectos que normalmente geram melhoria após a implantação do programa Seis Sigma: Operação e Cultura da Qualidade.

O teste de correlação demonstrou que a variável tempo de empresa apresenta correlação positiva com o constructo treinamento. Já a variável nível de formação em Seis Sigma apresentou correlação negativa Treinamento, Comunicação e Revisão e Operação. Dessa forma, pode-se verificar que as duas variáveis testadas apresentam correlação com parte dos constructos pesquisados.

O presente artigo contribuiu explorando os FCS em uma importante indústria alimentícia brasileira. Os resultados podem auxiliar empresas industriais que estão no processo de implantação do Seis Sigma, inserindo no seu planejamento de implantação ações para que os FCS sejam levados em consideração. Assim, os resultados esperados com a implantação de metodologia terão maior chance de serem alcançados e sustentados ao longo do tempo. Além disso, os resultados contribuem para outras pesquisas acadêmicas, pois o seu tratamento estatístico permite a comparação com resultados de pesquisa futuras que analisarem os FCS estudados.

Para trabalhos futuros, sugere-se a aplicação da pesquisa em indústrias alimentícias de forma a comparar os resultados obtidos com os desta pesquisa. Além disso, sugere-se a aplicação de múltiplos casos em empresas industriais para identificar como é feito o processo de implantação do Seis Sigma e quais os resultados obtidos em empresas que fizeram a implantação com o apoio dos FCS.

\section{REFERÊNCIAS}

ABIA. Associação Brasileira das Indústrias de Alimentos. Faturamento 2011, 2012. Disponível em: http://www.abia.org.br/vst/faturamento.pdf. Acesso em: 7 nov 2014. 
ANDERSSON, R.; ERIKSSON, H.; TORSTENSSON, H. Similarities and differences between TQM, six sigma and lean. The TQM Magazine, v. 18, n. 3, p. 282-296, 2006. http://dx.doi.org/10.1108/09544780610660004

BRUN, A. Critical success factors of Six Sigma implementations in Italian companies. International Journal of Production Economics, v. 131, n. 1, p. 158-164, 2011. http://dx.doi.org/10.1108/09544780210416702

CHAKRAVORTY, S. S. Six Sigma programs: An implementation model. International Journal of Production Economics, v. 119, n. 1, p. 1-16, 2009.

http://dx.doi.org/10.1016/j.ijpe.2009.01.003

CLETO, M. G.; QUINTEIRO, L. Gestão de projetos através do DMAIC: um estudo de caso na indústria automotiva. Revista Produção Online, v.11, n.1, p. 210-239, 2011. http://dx.doi.org/10.14488/1676-1901.v11i1.640

CRONBACH, L...Coefficient Alpha and the Internal Structure of Tests. Psycometrika, v.16, n.3, p. 297-335, 1951

DEVELLIS, R. F. Scale development: theory and applications, 2ed., Newbury Park, CA: Sage Publications, 2003.

DROHOMERETSKI, E.; GOUVEA DA COSTA, S. E.; PINHEIRO DE LIMA, E.; GARBUIO, P. A. R. Lean, Six Sigma and Lean Six Sigma: an analysis based on operations strategy. International Journal of Production Research, v.52, n.3, p. 804-824, 2014. http://dx.doi.org/10.1080/00207543.2013.842015

ECKES, G. A Revolução seis sigma: o método que levou a GE e outras empresas a transformar processos em lucro. Rio de Janeiro: Campus, 2001.

FIELD, A. Discovering Statistics Using SPSS. Newbury Park, CA: SAGE Publications, 2009.

FORZA, C. Survey research in operations management: a process based-perspective. International Journal of Operations \& Production Management, v. 22, n.2, p.152-194, 2002. http://dx.doi.org/10.1108/01443570210414310

HAIR JR., J. F.; ANDERSON, R. E.; TATHAM, R.; BLACK, W. C. Análise multivariada de Dados. 5. ed. Porto Alegre: Bookman, 2005.

HAN, C.; LEE, Y. Intelligent integrated plant operation system for six sigma. Annual Reviews in Control, v.26, p.27-43, 2002. http://dx.doi.org/10.1016/S1367-5788(02)80008-6

HENDERSON, H. K. M.; EVANS, J. R. Successful implementation of Six Sigma: benchmarking General Electric Company. Benchmarking: An International Journal, v. 7, n. 4, p.260-282, 2000. http://dx.doi.org/10.1108/14635770010378909

HINDO, B.; GROW, B. Six Sigma: so yesterday. Business Week, v. 1, jun., 2007. http://dx.doi.org/10.1016/j.orgdyn.2005.06.009

HUNG, H.; SUNG, M. Applying six sigma to manufacturing processes in the food industry to reduce quality cost. Scientific Research and Essays, v. 6, n. 3, p. 580-591, 2011.

http://dx.doi.org/10.5897/SRE10.823

Revista Produção Online, Florianópolis, SC, v. 16, n. 2, p. 475-498, abr./jun. 2016. 
KUMAR, S.; SOSNOSKI, M. Using DMAIC Six Sigma to systematically improve shopfloor production quality and costs. International Journal of Productivity and Performance Management, v. 58. n.3, p.254-273, 2009. http://dx.doi.org/10.1108/17410400910938850 KUMAR, S.; ANTONY, J.; TIWARI, M. K. Six Sigma implementation framework for SMEs - a roadmap to manage and sustain the change. International Journal of Production Research, v.49, n.18, p.5449-5467, 2011. http://dx.doi.org/10.1080/00207543.2011.563836

MAHANTI, R.; ANTONY, J. Six Sigma in the Indian software industry: some observations and results from a pilot survey." The TQM Journal, v. 21, n.6, p. 549-564, 2009. http://dx.doi.org/10.1108/17542730910995837

MALHOTRA, M. K.; GROVER, V. An assessment of survey research in POM: from constructs to theory. Journal of Operations Management, v.16, n.4, p. 407-425, 1998. http://dx.doi.org/10.1016/S0272-6963(98)00021-7

MARZAGÃO, D. S. L.; LOPES, A. P. V. B. V.; GOUVÊA, M. A.; CARVALHO, M. M. Fatores críticos de sucesso na implementação do programa Seis Sigma: uma revisão sistemática das pesquisas quantitativas. Revista Produção Online, SC, v.14, n. 2, p. 465-498, 2014. http://dx.doi.org/10.14488/1676-1901.v14i2.1348

MOOSA, K.; SAJID, S. Critical analysis of Six Sigma implementation. Total Quality Management \& Business Excellence, v.21, n.7, p.745-759, 2010. http://dx.doi.org/10.1080/14783363.2010.483100

NABHANI, F.; SHOKRI, A. Reducing the delivery lead time in a food distribution SME through the implementation of six sigma methodology. Journal of Manufacturing Technology Management, v.20, n.7, p.957-974, 2009. http://dx.doi.org/10.1108/17410380910984221

NÄSLUND, Dag. Lean, six sigma and lean sigma: fads or real process improvement methods? Business Process Management Journal, v.14, n.3, p. 269-287, 2008. http://dx.doi.org/10.1108/14637150810876634

NONTHALEERAK, P.; HENDRY, L. Six Sigma: literature review and key future research areas. International Journal of Six Sigma and Competitive Advantage, v. 2, n. 2, p. 105 38, 2006. http://dx.doi.org/10.1504/lJSSCA.2006.010111

NONTHALEERAK, P; HENDRY, L. Exploring the six sigma phenomenon using multiple case study evidence. International Journal of Operations \& Production Management, v.28, n.3, p.105-138, 2005. http://dx.doi.org/10.1108/01443570810856198

PANDE, P. S. Estratégia seis sigma. Rio de Janeiro: Qualitymark,2001.

PINTO, S.H.B.; CARVALHO, M.M.; HOO, L. L. Programa Seis Sigma: aspectos sinérgicos com outras abordagens de gerenciamento da qualidade. Revista Produção Online, v.9, n.1, p. 170-193, 2009. http://dx.doi.org/10.14488/1676-1901.v9i1.203

RODRIGUES, J. T. M. C.; WERNER, I . A gestão de pessoas contribuindo com o programa Seis Sigma: multi-casos de empresas instaladas no Rio Grande do Sul. Revista Produção Online. v.11, n.3, p.823-850, 2011. http://dx.doi.org/10.14488/1676-1901.v11i3.912 
SANTOS, A. B.; MARTINS, Manoel Fernando. Contribuições do Seis Sigma: estudos de caso em multinacionais. Production, v.20, n.1, p.42 - 53, 2010.

http://dx.doi.org/10.1590/S0103-65132010005000003

SHAHIN, A. Critical Success Factors: a comprehensive review. Proceedings of the International Conference on Problem Solving Strategies \& Techniques - PSST 2006, Tehran, 2006.

SIVAKUMAR, S; MUTHUSAMY, K. Critical Success Factors in Six Sigma

Implementation - a case study of mncs in malaysia. Proceedings of the 2011 IEEE ICQR, 536-540, 2011.

SNEE, R. D. Why Should Statisticians Pay Attention to Six Sigma?. Quality Progress, v. 9, p. $100-104,1990$.

SNEE, R. D. Impact of Six Sigma on quality engineering. Quality Engineering, v. 12, n.3, p. 9-14, 2000.

SU, Chao-ton. Improving service quality by capitalising on an integrated Lean Six Sigma methodology Tai-Lin Chiang Che-Ming Chang. Science, v.2, n.1, p. 1-22, 2006.

http://dx.doi.org/10.1504/IJSSCA.2006.009367

TRAD, S. Seis Sigma: fatores críticos de sucesso de sua implantação e impacto sobre desempenho organizacional. 2006. 177p. Dissertação (Mestrado em Administração) Universidade de São Paulo. São Paulo, 2006.

TRAD, S.; MAXIMIANO, A. C. A. Seis Sigma: fatores críticos de sucesso para sua implantação. Rev. adm. contemp., v.13, n.4, p.647-662, 2009.

http://dx.doi.org/10.1590/S1415-65552009000400008

VAN IWAARDEN, J.; VAN DER WIELE, T.; DALE, B. The Six Sigma improvement approach: a transnational comparison. International Journal of Production Research, v. 46, n. 23,

p. 6739-6758, 2008. http://dx.doi.org/10.1080/00207540802234050

YI-ZHONG, M. A.; YUE, G.; WANG, L.; SANGBOK. R. The Critical Success Factors of Six Sigma in China Manufacturing Industry. The Asian Journal on Quality, v.9, n.2, p. 39-56, 2011. http://dx.doi.org/10.1108/15982688200800016

ZAILANI, S.; SASTHRIYAR, S. Investigation on the Six Sigma Critical Success Factors. European Journal of Scientific Research, v.57, n.1, p. 124-132, 2011.

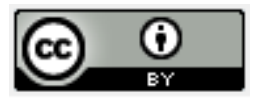

Artigo recebido em 08/12/2014 e aceito para publicação em 11/12/2014 DOI: http://dx.doi.org/ 10.14488/1676-1901.v16i1.1949 